\title{
Contents, Vol. 29, 1913
}

Inhalts -Verzeichnis.

Original-Arbeiten. Seite

Asmus, Eduard, Ueber die Bedeutung genauer Messungen bei

Augenmuskelvorlagerungen. Hierzu Taf. VII-VIII) 422, 515 Belenky-Raskin, Z., Zur Aetiologie

der phlyktänulären Augen-

entzündung 503

Brunzloiv, Beitrag zurAetiologie der chronischenErkrankungen

der tränenableitenden Wege 445

Clausen, W., Ueber Anwendung der Noviformsalbe in der

äußeren Augenheilkunde 295

Guny, F., Weitere Untersuchungen über den Zusammenhang

von Sehschärfe und Schießleistung der Infanterie . . .135

Czaplewski, E., Untersuchungen über Trachom 159

-, (II. Mitteilung.) (Hierzu Taf. II-V)267

Dalmer, M., Ueber einen Fall von Pseudo-Keratokonus . . 37 --·-, Ueber das Versagen der

Magnetextraktion bei posi-

tivem Sideroskopbefund

Elschnig, A., und R. v. Zeynek, Cataracta nigra 401

Erb, A., Lymphangiom der Bindehaut des Augapfels unter

dem Bilde eines Hämangioms

Fürth, 0. v. und V. Hanke, Studien über Quellungsvorgänge

am Auge 252

Guzmann, E., Ueber epibulbäre Tuberkulose' .34

Haudek, M., Ueber den Nachweis und die Lokalisation schwerer

Fremdkörper im Auge mittels der Böntgeustrahlen. . . 231 Hesse, R. und E. Phleps, Schichtstar und Tetanie .... 238 IgersTieimer, 3'., Ueber das Verhalten der Körpertemperatur

bei Erkrankungen des Auges 1

Isabolinsky, M. und W. Spassky, Zur Frage über den diagnostischen Wert der ,,Chlamydozoa”-Prowazek-Halberstädter beim Trachom 109

Junius, P., Bemerkungen zu den Mitteilungen von Professor

Czaplewski: Untersuchungen über Trachom 451

Kaelin-Benziger, Beiträge zur Behandlung der Stauungspapille, insbesondere bei Hirntumoren, durch Dekompressiv-(Palliativ-)Trepanation, mit temporärer extrakranieller Drainage eines Seitenventrikels .... 12, 138 Knapp, Paul, Beidseitige MakulaErkrankung nach Kurzschluß. (Hierzu Taf. IX) 440

Lerperger, O., Zwei Fälle von abnormer Innervation der Augen- 
muskel 565

$-\mathrm{IV}-$

Seite

Ollendorf, A., Die Kuhntsche Bindehautverwertung bei per-

forierenden Verletzungen 557

Rados, A., Ueber Plasmome der Bindehaut 125

Salzmann, M., Ueber den anämischen Fundus. (Hierzu

Taf. I) 30

Schejfels, 0., Zur Prioritätsírage betreífs der Kuhntschen

Bindehautplastik 299

Sepibus, J. v., Experimentelle Untersuchungen über die

Fluoreszenz der menschlichen Linse. (Hierzu Taf. VI) 407 Werncke, Th., Ueber die

Neurorezidive nach Salvarsanbe-

handlung 434

Wolff, H., Ueber neue ophthalmoskopische Untersuchungs-

methoden 216

Beriehte über die Deutsche ophthalmologische Literatur.

Experimentelle Pathologie und pathologische Anatomic.

Januar-Juni 1912. Von Prof. Dr. K. Stargardt in Kiel 39

Bericht über die Mikroorganismen. (II. Semester 1912.)

Von Priv.-Doz. Dr. Zade in Heidelberg 64

Anatomie des Auges. (I. Semester 1912.) Von Prof. Dr.

J. Sobotta in Würzburg 172

Die angeborenen Anomalien und Mißbildungen des Auges.(II. Semester 1912.) Von Priv.-Doz.

Dr. R. See]'elderin Leipzig 301

Motilitätsstörungen. (II. Semester 1912.) Voa Priv.-Doz.

Dr. C. H. Saltier in Gießen 307

Sympathische Ophthalmie. (II. Semester 1912.) Von Priv.-Doz. Dr. C. H. Saltier in Gießen 314

Spezielle $\mathrm{Pa}^{3} / 8$ hologie und Therapie. (I. und II. Semester 1912.)

Referent Dr. K. Schroder in Gera 317, 458, 569

Glaukom. (II. Semester 1912.) Von Priv.-Doz. Dr. C. H.

Saltier in Gießen 471

Beriehte über die ausländisehe ophthalmologische Literatur.

Amerikanische Literatur für das Jahr 1911. Von Prof.

R. C. Denig in New York 366

Bericht über die belgische Literatur (1910-1911). Von Dr.

H. Terlinck in Brüssel 166

Holländische ophthalmologische Literatur des Jahres 1912.

(II. Semester 1912.) Von Prof. Dr. W. Koster-Gzn in

Leiden und Dr. O. J. Schoute in Amsterdam 329

Russische ophthalmologische Literatur (I. Semester 1912.)

Von Priv.-Doz. Dr. Th. Werncke in Odessa 335

Skandinavische opthalmologische Literatur. (I. und II. Sem.

1911.) Von Priv.-Doz. Dr. E. Forsmark in Stockholm . 351

Gesellschafísberiehte. 


\section{Seite}

Berliner ophthalmologische Gesellschaft. Sitzungen vom

23. Januar, 28. November, 12. Dezember 1912 und

\section{Februar 1913 . 191,369, 479}

Vereinigung hessischer und Hessen-nassauischer Augenärzte.

Konstituierende Versammlung vom 29. Januar 1913 . . 371

Verein der Augenärzte von Ost- und Westpreußen. Sitzung am 1. Dezember 1912194

Bericht über die Sitzungen der Gesellschaft der Moskaueraugenärztlichen Gesellschaft.

Sitzungen vom 25. September, 30. Oktober und 27. November 1912

84

St. Petersburger Ophthalmologische Gesellschaft. Sitzungen vom 22. November, 20. Dezember 1912, vom 10. und

24. Januar, 14. und 28. März und 25. April 1913 . 373, 606Ophthalmologische Gesellschaft in Wien. Sitzungen vom

20. Mai, 17. Jani und 11. November, 16. Dezember 1912und 17. Februar 1913 ' $\quad 75$, 200, 480

Unfall- und Versicherungskunde $\quad 87,202,375,487,608$

Diagnostische Notizen $\quad 90,204,380,490,609$

Therapeutische Umschau 94, 206, 385, 492, 613

Insí umente 210

Aus den Grenzgebieten $\quad 390$

Buchanzeigen 98, 392

Literatur-Verzeichnis 103, 211, 395, 495, 616 